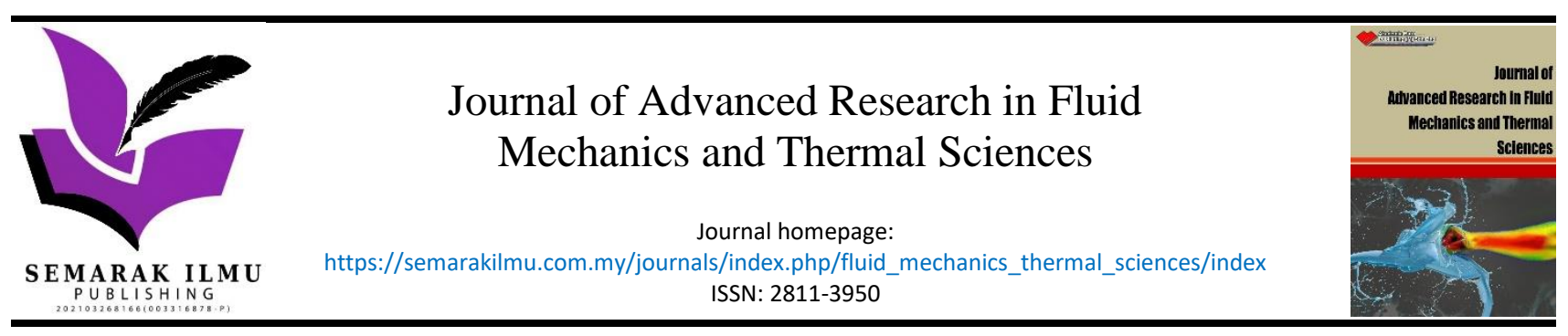

\title{
Modified Trailing Edge Impact on the Aerodynamic Performance of Wind Turbine Airfoil
}

\author{
Reham K. Jaafar ${ }^{1,}{ }^{*}$, M. A. Abdelrahman ${ }^{1}$, Mina G. Mourad ${ }^{1}$, Adnan A. Ateeq ${ }^{2}$, M. Moawed ${ }^{1}$ \\ Department of Mechanical Engineering, Benha University, Qalubia 13518, Egypt \\ Technical College, Southern Technical University, Basra 61006, Iraq
}

\section{ARTICLE INFO}

\section{Article history:}

Received 8 November 2021

Received in revised form 9 January 2022

Accepted 15 January 2022

Available online 27 January 2022

\section{Keywords:}

CFD; wind turbine airfoil; lift/drag ratio; lift coefficient

\section{ABSTRACT}

One of the most important factors affecting wind turbine performance is the airfoil. The impact of the NACA 0012 trailing edge design on airfoil performance is investigated numerically in this paper. Computational fluid dynamics calculations are used to design and simulate the airfoils. The thick trailing edge is inclined to various angles to achieve further improvement in the lift/drag ratio and lift coefficient. The results reveal that, when compared with baseline airfoil, all the designed airfoils demonstrated higher lift coefficients. The lift coefficient increases with the angle of the inclined trailing edge. The maximum lift coefficient improvement inclined airfoil is $74 \%$. In addition, the lift/drag ratio increases with the increase of the inclined angle, and the maximum improvement ratio reaches $39.495 \%$ for the inclined airfoil with $\theta=15$. Any further increase in the inclined angle decreases the lift/drag ratio as a result of drag increase. This study contributes toward the design of efficient wind turbine airfoils.

\section{Introduction}

With the increasing environmental pollution from fossil fuels, clean energy sources have become extremely important [1]. Among various systems of electrical energy generation, wind energy has acquired significant attention and become a major contributor in the world due to its availability and low cost [2]. Thousands of wind turbine plants have been built around the world and are used to supply electrical power to local electrical networks [3]. According to the last Global Wind Energy report, China has been able to install several wind power plants that, combined, produce $23 \mathrm{GW}$ of energy every year [3].

The blades are the main part of the wind turbine, in which kinetic energy is converted to useful power. Wind turbine performance depends considerably on the design of the blades, and in particular, the geometry of the airfoil. The airfoil is the core element of a blade [4]-[6] and it can also be used in many engineering applications [7]. Two of the most important factors that impact the performance of the airfoil are the lift/drag ratio $(\mathrm{Cl} / \mathrm{Cd})$ and lift coefficient. The desired wind turbine

\footnotetext{
* Corresponding author.

E-mail address: reham.kadhim@stu.edu.iq
} 
should have an airfoil with a high lift/drag ratio $(\mathrm{Cl} / \mathrm{Cd})$ and lift coefficient [9]. The approaches that have been utilized to enhance the lift/drag ratio $(\mathrm{Cl} / \mathrm{Cd})$ and lift coefficient are classified into two types: active and passive systems. In the active system, an external power source such as plasma actuators [10], synthetic jets [11], or microtabs [12] is usually utilized to control the boundary layer and subsequently improve the aerodynamic forces. In contrast, the passive system does not require an external power source and is, generally, simpler when compared with an active system. Therefore, it has been used by various researchers to enhance the performance of existing airfoils. For instance, Taylor et al., [13] and Fouatih et al., [14] used a vortex generator that consists of a row of blades or airfoils to control the boundary layer. The vortex generator was placed slightly higher than the boundary layer and was set at an angle against the oncoming flow. The presence of this device markedly improved the wind turbine performance by forming vortices that propagated downstream to improve the free flow mixing with the boundary layer; consequently, the boundary layer stayed linked to the airfoil. Dane Gurney [15] inserted a flat plate (known as the Gurney flap) at the end of the airfoil, perpendicularly to the suction side. The inserted plate led to a noticeable increase in lift coefficient $[16,17]$. The slotted airfoil was first introduced by Handley Page [18], in which the addition of slots to the airfoil controls the flow around the airfoil to improve the lift coefficient [3], [19]. Zhuang et al., [20] and Jawahar et al., [21] have examined the effect of a morphed trailing edge airfoil (flap airfoil) with various angles from the chord line on aerodynamic performance by using a computational fluid dynamic (CFD), with the results showing that the lift coefficient and lift/drag improve. A numerical computation performed by Ma et al., [22] to analyze the effects of relative thickness and its position on the aerodynamic performance of an airfoil can also be used to improve the lift coefficient.

In the aforementioned studies, different approaches have been studied individually to improve the lift coefficient and lift/drag ratio of the airfoil in the passive system. Other researchers have used a combination of two methods to optimize the lift/drag ratio and lift coefficient. For instance, $\mathrm{Xu}$ et al., [23] studied different thick trailing edges with asymmetrical blunt trailing-edge airfoils. Their results show that the thickness position from the leading edge could be considered as a factor to modify the blunt airfoil and upgrade the lift/drag ratio. Gao et. al [24] and Kundu et al., [25] studied a vortex generator with a blunt and thick trailing-edge airfoil, respectively. Gamiz et al., [26] utilized Gurney flaps with microtabs to improve the lift/drag ratio for all angles of attack.

However, there is little research focusing on the combination of two approaches to improve the lift/drag ratio and lift coefficient of a wind turbine. The effects of the thickness and morphed trailing edge modification on the airfoil have only been studied individually. It is important, however, to probe the aerodynamic performance of airfoils with thickness and morphed trailing edge modifications to improve the design of a wind turbine blade. Therefore, herein, we utilize these two approaches to modify the airfoil and study the effects on the lift/drag ratio and lift coefficient. To enhance the aerodynamic performance, NACA 0012 is used for the simulation. The thick trailing edge was inclined (morphed) to various angles (from $5^{\circ}$ to $40^{\circ}$ ) to achieve further improvement in the lift/drag ratio and lift coefficient. The results revealed that inclining the thick trailing edge at 150 resulted in superior performance.

\section{Numerical Modeling}

\subsection{Physical Model}

The impact of a two-dimensional airfoil's trailing edge on its aerodynamic performance is investigated. The inclined trailing edge is implemented on the 2D modified NACA 0012 airfoil of with the thickness ratio of the trailing edge $2 \% c$, according to our previous study [27], as shown in Figure 
1. The angles of inclined trailing edge method are set to $5,10,15,20,25,30,35$ and 40 . The angles are measured between the chord of the airfoil and the trailing edge. The chord length of the airfoil remains constant in all cases. The total length of the chord is $0.2 \mathrm{~m}$. Small scale wind turbines are a concerned in the present study.

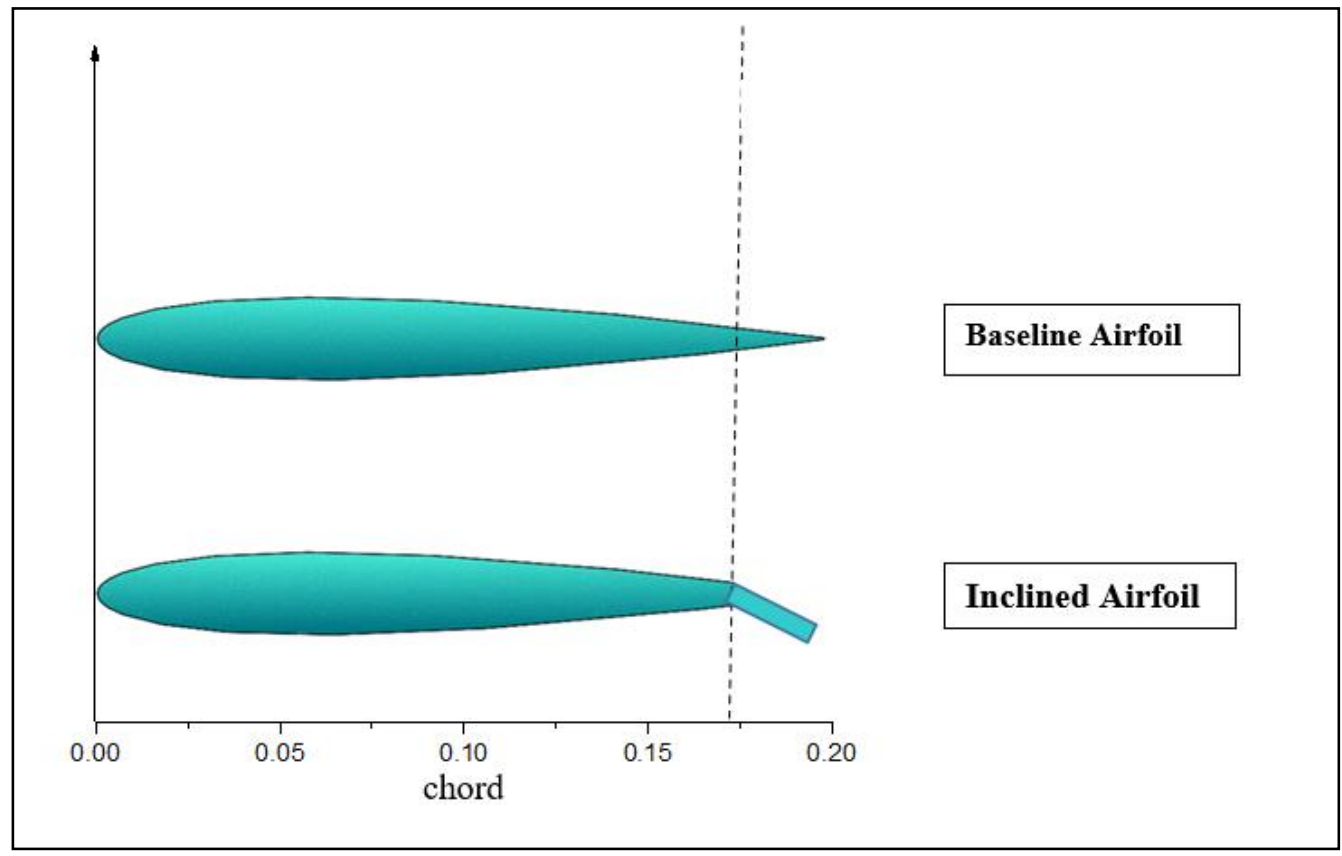

Fig. 1. NACA 0012 with and without inclined trailing edge

\subsection{Computational Domain and Boundary Conditions}

As illustrated in Figure 2, the $\mathrm{C}-\mathrm{H}$ type is chosen for the computational domain. To reject the effect of the size domain, the simulation domain has been carefully selected 20 times from the chord of the airfoil for high accuracy $[28,29]$. Hence, in this study, the simulation domain has been utilized 25 from the airfoil chord and generated using ANSYS 19. The length of the airfoil chord is $0.2 \mathrm{~m}$ as mentioned before. For all cases, the following general boundary conditions are applied: At free flow conditions, the upstream and side boundary conditions are modeled as velocity inlets. The downstream boundary is specified as a pressure outlet, and the velocity is assumed to be uniform about $8 \mathrm{~m} / \mathrm{s}$. Furthermore, the airfoil's surface is treated as a non-slip wall. The lift and drag coefficients equations, which were utilized to analyze the data of the baseline and inclined airfoil, are as follows:

$$
\begin{aligned}
& C_{L}=\frac{L}{0.5 \rho U C} \\
& C_{D}=\frac{D}{0.5 \rho U C} \\
& \mathrm{~L}=\mathrm{N} \cos (\alpha)-\mathrm{A} \sin (\alpha) \\
& \mathrm{D}=\mathrm{N} \sin (\alpha)+\mathrm{A} \cos (\alpha)
\end{aligned}
$$


The lift and drag ratios are used to calculate aerodynamic efficiency. The pressure coefficient is also determined by:

$C_{p}=\frac{P}{0.5 \rho U C}$

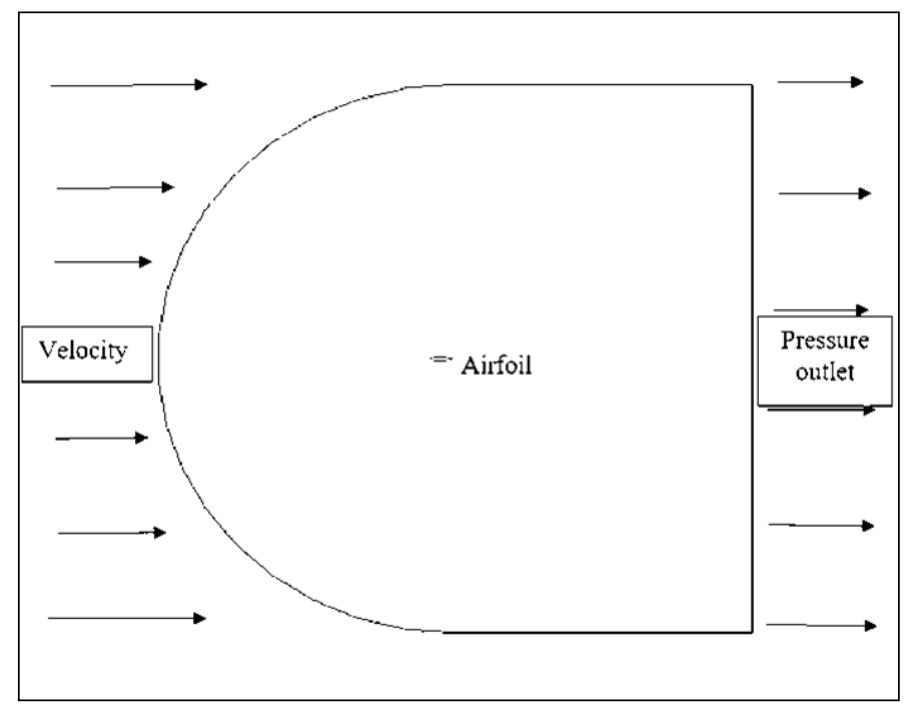

Fig. 2. The domain of computational

\subsection{Numerical Simulation}

The calculations in this study are done using the steady state numerical simulation method. Eleni et al., [25] demonstrated that the kw- SST model is appropriate for NACA 0012 airfoil design and provides accurate analyses and vortex at all angles of attack. Furthermore, according to references $[28,30,31], k$ SST simulated the boundary layers along the airfoil's wall, which is necessary for capturing accurate results through numerical computations. Based on these researches, the KW-SST model is selected for all domains in the current work for both airfoils with and without inclined trailing edge. The type of coupling algorithm is used to solve the equations of pressure-velocity coupling and the second-order upwind is selected with double-precision is used to calculate the governing equations including the continuity, momentum, and turbulence equations. The Iteration value of the calculations is stopped at $10^{-6}$ in the current study. The governing equations for the conservation of mass and momentum can be written as:

Continuity equation:

$\frac{\partial u}{\partial x}+\frac{\partial v}{\partial x}=0$

Two-dimensional incompressible Navier-Stokes equations:

$$
\begin{aligned}
& \frac{\partial u}{\partial t}+\mathrm{u} \frac{\partial u}{\partial x}+\mathrm{U} \frac{\partial u}{\partial x}=\frac{1}{\rho} \frac{\partial p}{\partial x}+\frac{\mu}{\rho}\left(\frac{\partial^{2} u}{\partial x^{2}}+\frac{\partial^{2} u}{\partial y^{2}}\right) \\
& \frac{\partial v}{\partial t}+\mathrm{u} \frac{\partial v}{\partial x}+\mathrm{U} \frac{\partial v}{\partial x}=\frac{1}{\rho} \frac{\partial p}{\partial y}+\frac{\mu}{\rho}\left(\frac{\partial^{2} v}{\partial x^{2}}+\frac{\partial^{2} v}{\partial y^{2}}\right)
\end{aligned}
$$


where

$\rho=1.225 \mathrm{~kg} / \mathrm{m}^{3}$

$\mu=1.7894 * 10^{5} \mathrm{~kg} /(\mathrm{m} . \mathrm{s})$

The turbulent kinetic energy transport model equations of $k$ and $\omega$ can be stated by the following, respectively:

$\frac{\partial(\rho \mathrm{k})}{\partial t}+\frac{\partial\left(\rho u_{j} k\right)}{\partial x_{j}}=p-6^{*} \rho \omega \dot{k}+\frac{\partial}{\partial x_{j}}\left[\left(\sigma_{k} \mu_{t}+\mu\right) \frac{\partial k}{\partial x_{j}}\right]$

$\frac{\partial \rho \dot{\omega}}{\partial t}+\frac{\partial\left(\rho u_{j} \dot{\omega}\right)}{\partial x_{j}}=\frac{\gamma}{V_{t}} P-\rho B \dot{\omega}^{2}+\frac{\partial}{\partial x_{j}}\left[\left(\sigma_{\dot{\omega}} \mu_{t}+\mu\right) \frac{\partial \dot{\omega}}{\partial x_{j}}\right]+2\left(1-\mathrm{F}_{1}\right) \sigma_{\dot{\omega}^{2}}\left(\frac{\rho}{\dot{\omega}}\right)\left(\frac{\partial K}{\partial x_{j}}\right)\left(\frac{\partial \dot{\omega}}{\partial x_{j}}\right)$

where

$S_{i j}=0.5\left(\frac{\partial u_{j}}{\partial x_{i}}+\frac{\partial u_{i}}{\partial x_{j}}\right), P=\tau_{i j} \frac{\partial u_{i}}{\partial x_{j}}, \tau_{i j}=\mu_{t}\left(2 S_{i j}-\frac{2}{3} \frac{\partial u_{k}}{\partial x_{k}} S_{i j}\right)-\frac{2}{3} \partial k S_{i j}$

\subsection{Mesh Independence Test}

The grid independence test is generally performed to ensure that the results do not depend on the number of elements [32]. The grid independence study is performed for the NACA 0012 airfoil at an attack angle of 40 and $\mathrm{Re}=3 \times 10^{6}$ by using ANSYS-R19-ICEM. The structured mesh is utilized in this study because its heigh accuracy in predicting the experimental data [33]. Table 1 shows the standard parameters of the mesh quality; it is clear that our results fall within the scope of standard mesh quality in the ICEM software [34]. The hexahedral mesh type is used as shown in Figure 3. The range of the dimensionless distance of the wall $(\mathrm{Y}+)$ is smaller than (1), which is necessary to figure out more information about the flow of the boundary layer over the airfoil surface. Three different nodes number are compared in size: coarse, medium, and large $(63,644,119,909$, and 181,739, respectively). The grids are taken when the magnitude of the lift and drag coefficients stops significantly varying with an increase of node number. Figure 4 shows that convergence occurs when the grids number 119,909 , and so this number is used for all the current cases of airfoil design flow simulations. The relative error value is $7 \%$.

Table 1

The values of mesh quality

\begin{tabular}{lll}
\hline Parameter & Standard Mesh Quality [34] & Present Work \\
\hline Angle & Greater than 18 & 59 \\
Minimum determinant & Greater than 0.2 & 0.8 \\
\hline
\end{tabular}




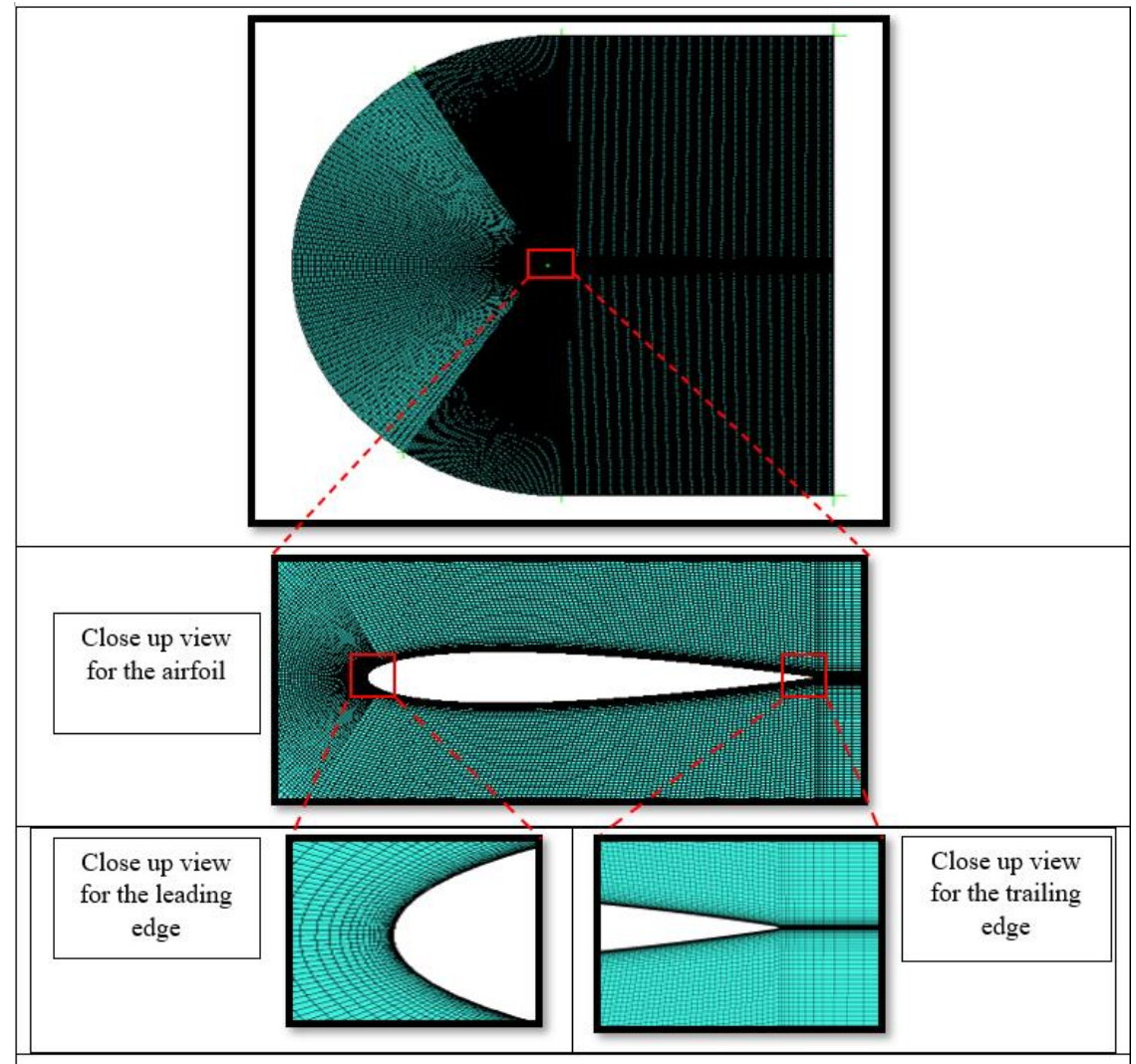

Fig. 3. Distribution of mesh around the airfoil

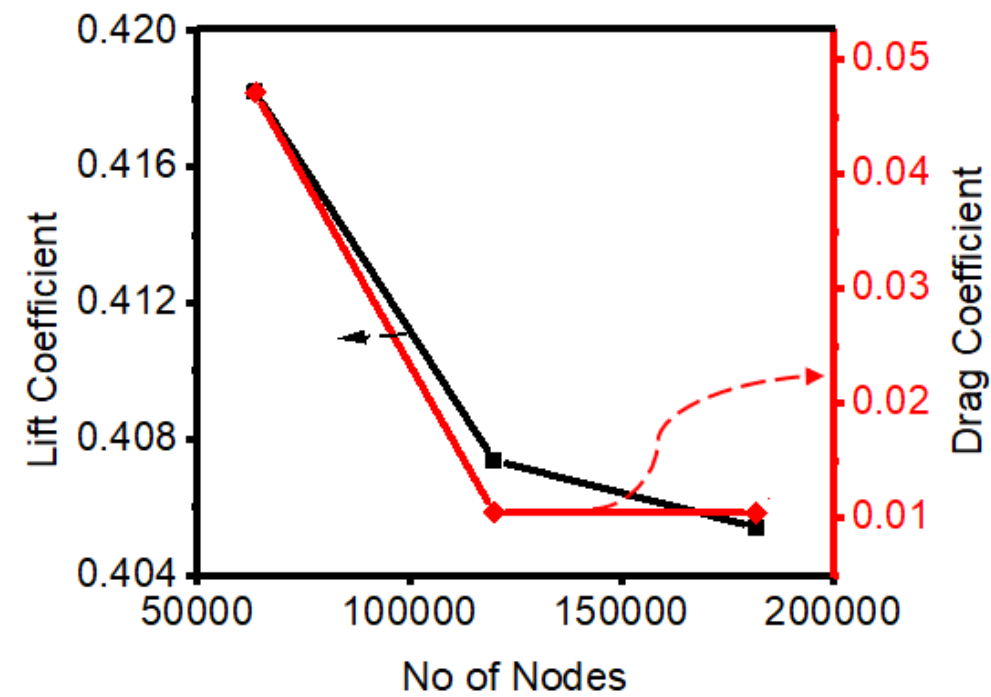

Fig. 4. Comparisons of three different numbers of grids at $\alpha=4^{\circ}$

\section{Results and Discussion}

\subsection{Aerodynamic Performance of Airfoils with Inclined Thick Trailing Edge at Different Angles}

In this section, the trailing edge of the optimized thick trailing edge is inclined by various angles $(\theta)-5^{\circ}, 10^{\circ}, 15^{\circ}, 20^{\circ}, 25^{\circ}, 30^{\circ}, 35^{\circ}$, and $40^{\circ}$-as mentioned before, and the effects of these changes on lift/drag ratio and lift coefficient are studied. Figure 5(a) shows the results for the lift and drag coefficients. The influence of the inclined thick trailing edge is to considerably enhance the lift 
coefficient. Compared with the baseline airfoil and smooth thick airfoil, the maximum lift coefficient is gradually improved with the increase of the angle of the inclined trailing edge. No matter what the angle is, the lift coefficient is higher than that of the baseline airfoil for all angles of attack. Besides, Figure 5(a) also indicates that the optimum lift coefficient for the inclined airfoil occurs at $\alpha=10^{\circ}$ and is smaller than the stall thick airfoil angle. The maximum lift coefficient value is 1.44 for the inclined airfoil of $\theta=40^{\circ}$ and is increased by $74 \%$. The drag coefficients versus the angles of attack are also shown in Figure 5(a). The drag coefficient slightly increases with the inclined angle when the $\alpha$ is between $1^{\circ}$ and $8^{\circ}$, however, at an $\alpha$ above $8^{\circ}$, a considerable increase in the drag coefficient is observed. In Figure 5 (b), the lift/drag ratios for the inclined airfoils are greater than that of the baseline airfoil. The lift/drag ratio increases with the angle of the inclined trailing edge. The maximum lift/drag ratio is achieved for the inclined airfoil of $\theta=15^{\circ}$ and is increased by $39.495 \%$. After that, the lift/drag ratio decreases with the enlargement of the angle of the inclined trailing edge until it reaches the minimum value at inclined airfoil of $\theta=40^{\circ}$. The lift/drag ratio of inclined airfoil of $\theta=40^{\circ}$ is approximately equal to that of the baseline airfoil. Because of the rate of increase, the drag coefficient begins to grow larger than the rate of increase of the lift coefficient, and then the lift/drag ratio drops significantly to that of the baseline airfoil. Figure 6 shows the pressure coefficient of the baseline airfoil and inclined airfoils. The figure illustrates that as the angle of inclined trailing edge airfoil increases, the difference in the pressure coefficient increases. The optimum pressure coefficient is reached for the inclined airfoil of $\theta=40$, and this, in turn, leads to an increase in the lift coefficient. The increase of the trailing edge angle $(\theta)$ causes an increase in trailed vortices formation, and this, consequently, increases the drag coefficient due to the increase in the negative pressure behind the trailing edge, as shown in Figure 7.

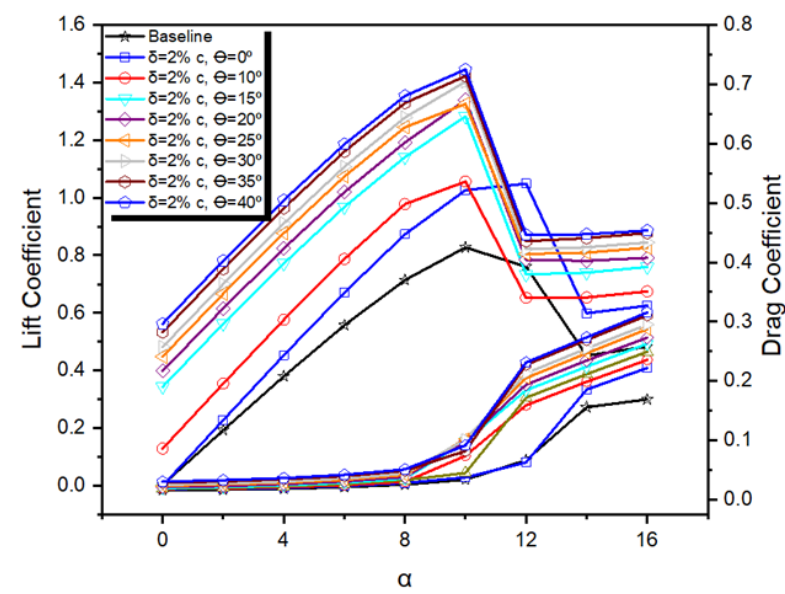

(a) Lift and drag coefficients

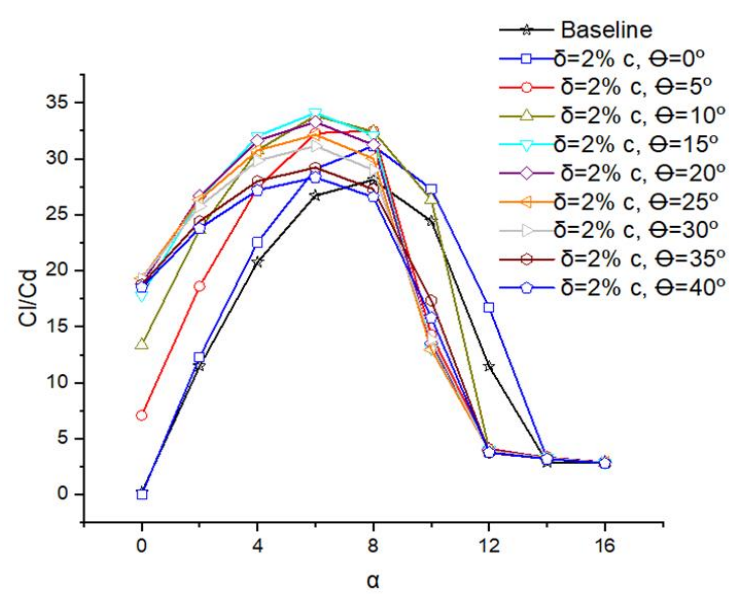

(b) Lift/drag ratio

Fig. 5. Aerodynamic coefficients for the baseline airfoil and inclined airfoils with angles of attack 


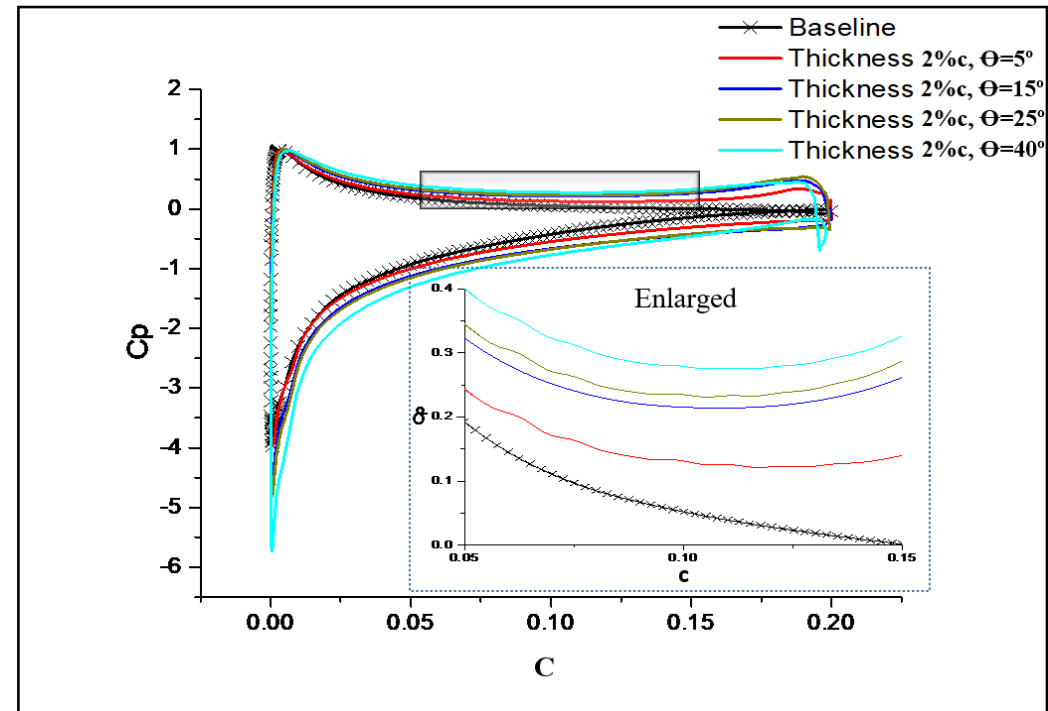

Fig. 6. Comparison of pressure coefficient over an airfoil with and without modification at $\alpha=10^{\circ}$.
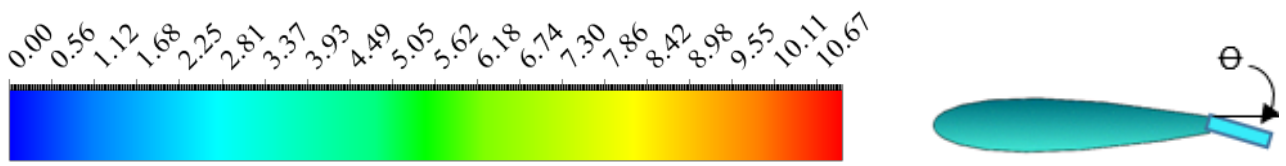

Velocity
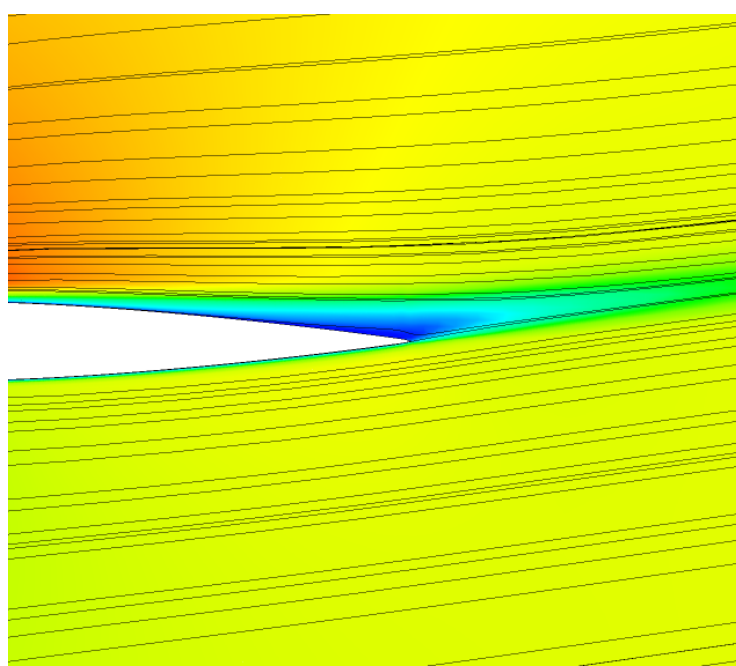

(a) Baseline airfoil $\left[\mathrm{m} \mathrm{s}^{\wedge}-1\right]$

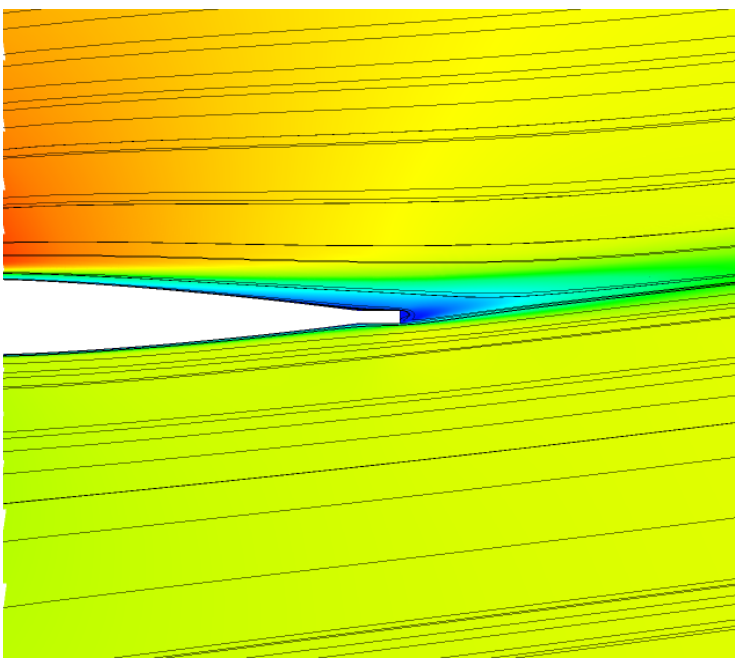

(b) Inclined airfoil with $\delta=2 \% c$ and $\theta=0^{\circ}$ 


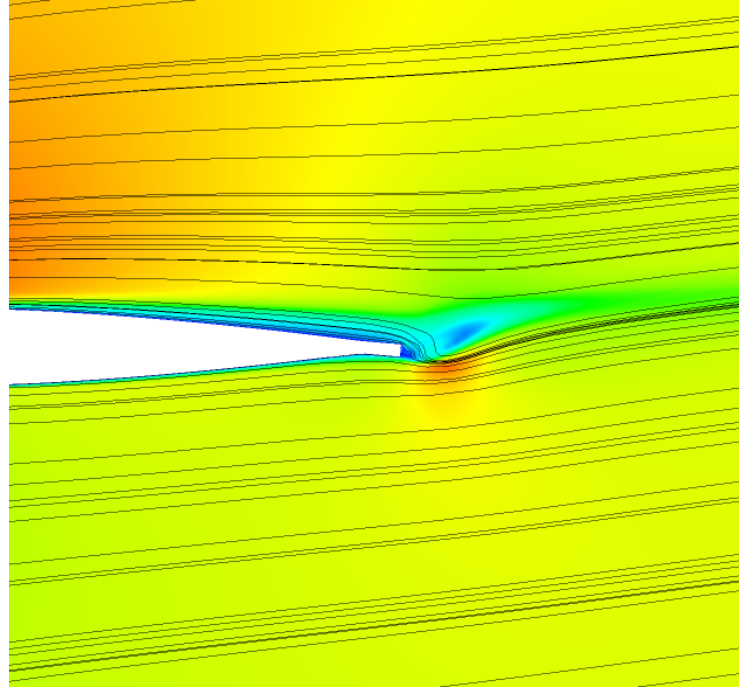

(c) Inclined airfoil with $\delta=2 \% \mathrm{c}$ and $\theta=50$

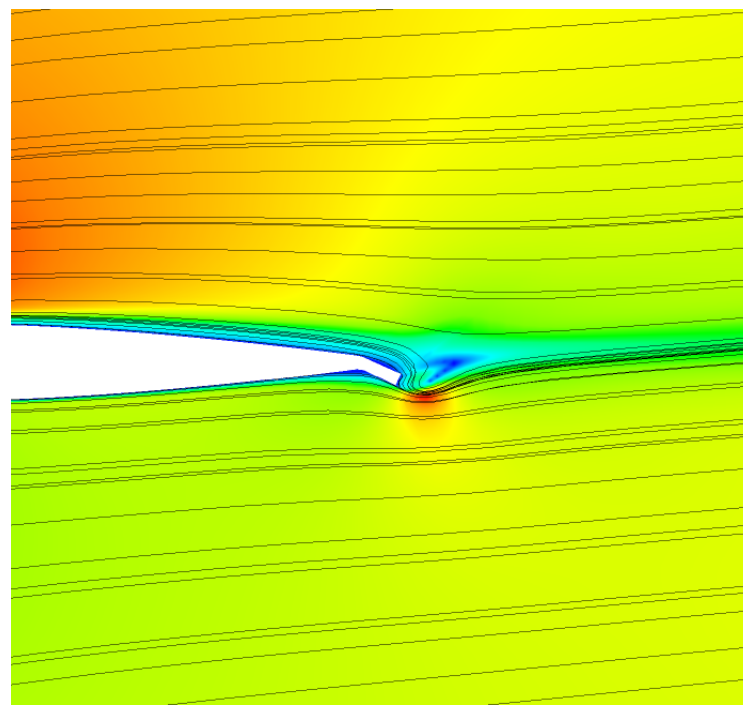

(e) Inclined airfoil with $\delta=2 \% c$ and $\theta=25$ 을

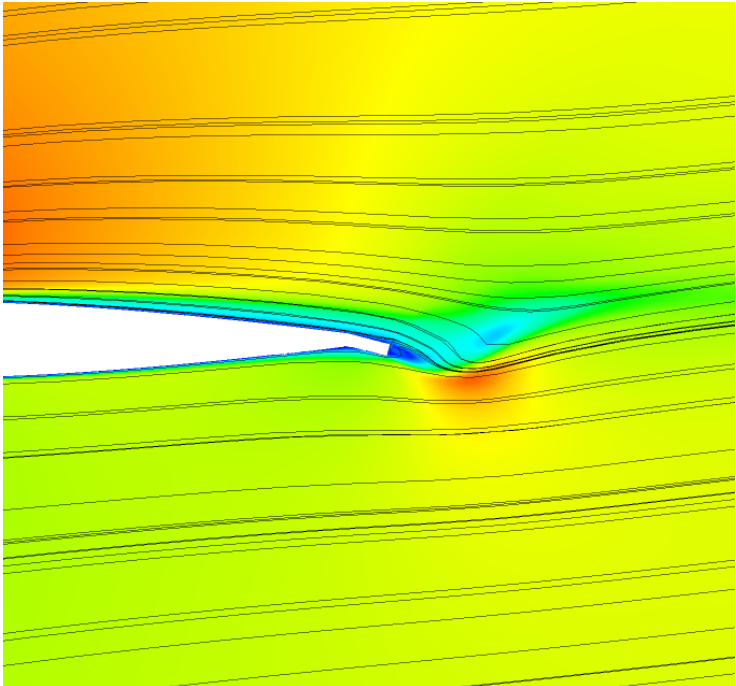

(d) Inclined airfoil with $\delta=2 \% \mathrm{c}$ and $\theta=15$

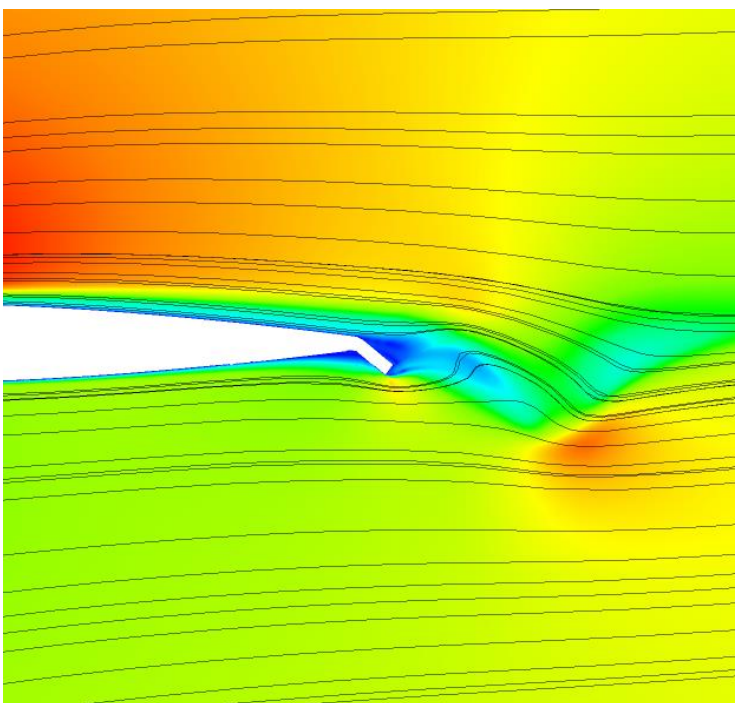

(f) Inclined airfoil with $\delta=2 \% \mathrm{c}$ and $\theta=40$ -

Fig. 7. Velocity contours and streamlines of the airfoils at $\alpha=10$ 을

As seen in the above method, the angle of inclined trailing edge stops at $=40^{\circ}$ as the lift $/ \mathrm{drag}$ ratio of these airfoils decreases and becomes too near to the lift/drag ratio of the baseline airfoil.

Table 2 summarizes the lift coefficient values and lift/drag ratios for all airfoils. The inclined airfoil with the best performance is highlighted. Based on the previous results, the case of the inclined airfoil with $\theta$ of $15^{\circ}$ is the best case in this study for improving the lift/drag ratio. Based on lift coefficient improvement, the case of the inclined airfoil with $\theta=40^{\circ}$ is the best airfoil when compared with other studied airfoils. 


\section{Table 2}

Comparisons of maximum values of lift coefficients and lift/drag ratios for baseline airfoil and thick airfoils

\begin{tabular}{|c|c|c|c|c|}
\hline $\begin{array}{l}\text { Percentage of } \\
\text { Modification }\end{array}$ & $\alpha$ & $\begin{array}{l}\text { Optimum Lift } \\
\text { Coefficient }\end{array}$ & $\alpha$ & Optimum Lift/Drag Ratio \\
\hline $\begin{array}{l}\text { Baseline Airfoil } \\
\text { Inclined Airfoil }\end{array}$ & 10 & 0.8278 & 8 & 28.1198 \\
\hline$\delta=2 \% c, \theta=50$ & 10 & 1.0563 & 8 & 32.4928 \\
\hline$\delta=2 \% c, \theta=100$ & 10 & 1.1816 & 6 & 33.8398 \\
\hline$\delta=2 \% c, \theta=150$ & 10 & 1.2829 & 6 & 34.0957 \\
\hline$\delta=2 \% c, \theta=20$ o & 10 & 1.3396 & 6 & 33.2791 \\
\hline$\delta=2 \% c, \theta=250$ & 10 & 1.3262 & 6 & 32.1454 \\
\hline$\delta=2 \% c, \theta=30$ - & 10 & 1.4036 & 6 & 31.1462 \\
\hline$\delta=2 \% c, \theta=35$ 은 & 10 & 1.4226 & 6 & 29.2188 \\
\hline$\delta=2 \% c, \theta=40$ 요 & 10 & 1.4455 & 6 & 28.3280 \\
\hline
\end{tabular}

\section{Conclusions}

This paper numerically investigates the effect of various inclined of thick trailing edge on the aerodynamic performance improvement by Computational fluid dynamics with the $k-\omega$ SST turbulence model. Based on our observation, the results are summarized as follows:

i. The lift coefficient increases with the increase in the inclining angle. The optimum lift coefficient improvement is achieved for the inclined airfoil with $\theta=40$, increasing by $74 \%$.

ii. The drag coefficient also increases with the inclined angle increases.

iii. The lift/drag ratio increases with the increase of the inclined angle, and the maximum improvement ratio reaches $39.495 \%$ for the inclined airfoil with $\theta=15 \circ$. Any further increase in the inclined angle decreases the lift/drag ratio as a result of drag increase.

To conclude, the inclined airfoil with $\theta=15$ o results in the highest lift/drag ratio (39.495\%) of all the studied airfoils.

\section{Acknowledgement}

The stimulating discussion with Dr. Ahmed Al Mayyahi at Kansas State University-USA and Prof. Dr M.F. Abd Rabbo at Benha university-Egypt prior to the initiation of this study and their constructive comments and editing to the final version of this manuscript.

\section{References}

[1] Varol, Asaf, Cumali İlkılıç, and Yasin Varol. "Increasing the efficiency of wind turbines." Journal of Wind Engineering and Industrial Aerodynamics 89, no. 9 (2001): 809-815. https://doi.org/10.1016/S0167-6105(01)00069-1

[2] Sewucipto, Sanjaya, and Triyogi Yuwono. "The Influence of Upstream Installation of D-53 Type Cylinder on the Performance of Savonius Turbine." Journal of Advanced Research in Experimental Fluid Mechanics and Heat Transfer 3, no. 1 (2021): 36-47.

[3] Belamadi, Riyadh, Abdelouaheb Djemili, Adrian Ilinca, and Ramzi Mdouki. "Aerodynamic performance analysis of slotted airfoils for application to wind turbine blades." Journal of wind engineering and industrial aerodynamics 151 (2016): 79-99. https://doi.org/10.1016/i.jweia.2016.01.011

[4] Masud, M. H., Naim-Ul-Hasan, Amit Md Estiaque Arefin, and Mohammad UH Joardder. "Design modification of airfoil by integrating sinusoidal leading edge and dimpled surface." In AIP Conference Proceedings, vol. 1851, no. 1, p. 020048. AIP Publishing LLC, 2017. https://doi.org/10.1063/1.4984677

[5] Tirandaz, M. Rasoul, and Abdolrahim Rezaeiha. "Effect of airfoil shape on power performance of vertical axis wind turbines in dynamic stall: Symmetric Airfoils." Renewable Energy 173 (2021): $422-441$. https://doi.org/10.1016/i.renene.2021.03.142

[6] Sharma, Sparsh, Thomas F. Geyer, and Jens Giesler. "Effect of geometric parameters on the noise generated by rod-airfoil configuration." Applied Acoustics 177 (2021): 107908. https://doi.org/10.1016/i.apacoust.2021.107908 
[7] Samion, Siti Ruhliah Lizarose, and Mohamed Sukri Mat Ali. "Aerodynamic noise measurement in anechoic wind tunnel of rod-airfoil with leading edge serrations." Journal of Advanced Research in Fluid Mechanics and Thermal Sciences 47, no. 1 (2018): 97-107.

[8] Samion, Siti Ruhliah Lizarose, Mohamed Sukri Mat Ali, and Con Doolan. "AERODYNAMIC NOISE MEASUREMENT OF ROD-AIRFOIL WITH LEADING EDGE SERRATIONS IN ANECHOIC WIND TUNNEL."

[9] Ju, Y. P., and C. H. Zhang. "Multi-point robust design optimization of wind turbine airfoil under geometric uncertainty." Proceedings of the Institution of Mechanical Engineers, Part A: Journal of Power and Energy 226, no. 2 (2012): 245-261. https://doi.org/10.1177/0957650911426540

[10] Ebrahimi, Abbas, and Mohammadreza Movahhedi. "Power improvement of NREL 5-MW wind turbine using multiDBD plasma actuators." Energy Conversion and Management $146 \quad$ (2017): 96-106. https://doi.org/10.1016/i.enconman.2017.05.019

[11] Taylor, Keith, Chia Min Leong, and Michael Amitay. "Load control on a dynamically pitching finite span wind turbine blade using synthetic jets." Wind Energy 18, no. 10 (2015): 1759-1775. https://doi.org/10.1002/we.1789

[12] Tsai, Kuo-Chang, Cheng-Tang Pan, Aubryn M. Cooperman, Scott J. Johnson, and C. P. Van Dam. "An innovative design of a microtab deployment mechanism for active aerodynamic load control." Energies 8, no. 6 (2015): 58855897. https://doi.org/10.3390/en8065885

[13] Mohamed, Omar S., Ahmed A. Ibrahim, Ahmed K. Etman, Amr A. Abdelfatah, and Ahmed MR Elbaz. "Numerical investigation of Darrieus wind turbine with slotted airfoil blades." Energy Conversion and Management: X 5 (2020): 100026. https://doi.org/10.1016/i.ecmx.2019.100026

[14] Fouatih, Omar Madani, Marc Medale, Omar Imine, and Bachir Imine. "Design optimization of the aerodynamic passive flow control on NACA 4415 airfoil using vortex generators." European Journal of Mechanics-B/Fluids 56 (2016): 82-96. https://doi.org/10.1016/j.euromechflu.2015.11.006

[15] Houghton, Edward Lewis, and Peter William Carpenter. Aerodynamics for engineering students. Elsevier, 2003.

[16] Kumar, P. Madhan, and Abdus Samad. "Introducing Gurney flap to Wells turbine blade and performance analysis with OpenFOAM." Ocean Engineering 187 (2019): 106212. https://doi.org/10.1016/j.oceaneng.2019.106212

[17] Hao, Li-Shu, and Yong-Wei Gao. "Effect of Gurney flap geometry on a S809 airfoil." International Journal of Aerospace Engineering 2019 (2019). https://doi.org/10.1155/2019/9875968

[18] Francis, Parker Humphrey. "Variable-camber rib for aeroplane-wings." U.S. Patent 1,341,758, issued June 1, 1920.

[19] Coder, James G., and Dan M. Somers. "Design of a slotted, natural-laminar-flow airfoil for commercial transport applications." Aerospace Science and Technology 106 (2020): 106217. https://doi.org/10.1016/i.ast.2020.106217

[20] Zhuang, Chen, Gang Yang, Yawei Zhu, and Dean Hu. "Effect of morphed trailing-edge flap on aerodynamic load control for a wind turbine blade section." Renewable Energy 148 (2020): 964-974. https://doi.org/10.1016/j.renene.2019.10.082

[21] Jawahar, Hasan Kamliya, Qing Ai, and Mahdi Azarpeyvand. "Experimental and numerical investigation of aerodynamic performance for airfoils with morphed trailing edges." Renewable Energy 127 (2018): 355-367. https://doi.org/10.1016/j.renene.2018.04.066

[22] Ma, Dongli, Yanping Zhao, Yuhang Qiao, and Guanxiong Li. "Effects of relative thickness on aerodynamic characteristics of airfoil at a low Reynolds number." Chinese Journal of Aeronautics 28, no. 4 (2015): 1003-1015. https://doi.org/10.1016/i.cja.2015.05.012

[23] Zhang, Xu, Wei Li, and Hailong Liu. "Numerical simulation of the effect of relative thickness on aerodynamic performance improvement of asymmetrical blunt trailing-edge modification." Renewable Energy 80 (2015): 489497. https://doi.org/10.1016/i.renene.2015.02.038

[24] Gao, Linyue, Hui Zhang, Yongqian Liu, and Shuang Han. "Effects of vortex generators on a blunt trailing-edge airfoil for wind turbines." Renewable Energy 76 (2015): 303-311. https://doi.org/10.1016/j.renene.2014.11.043

[25] Kundu, Parikshit, Arunjyoti Sarkar, and Vishwanath Nagarajan. "Improvement of performance of S1210 hydrofoil with vortex generators and modified trailing edge." Renewable Energy 142 (2019): 643-657. https://doi.org/10.1016/i.renene.2019.04.148

[26] Fernandez-Gamiz, Unai, Macarena Gomez-Mármol, and Tomas Chacón-Rebollo. "Computational modeling of gurney flaps and microtabs by POD method." Energies 11, no. 8 (2018): 2091. https://doi.org/10.3390/en11082091

[27] Jaafar, Reham K., Mina G. Mourad, and Adnan A. Ateeq. "Effect of Thick Trailing Edge on the Aerodynamic Performance of NACA0012 Airfoil." Design Engineering (2021): 17236-17247.

[28] Mohamed, M. H., A. M. Ali, and A. A. Hafiz. "CFD analysis for H-rotor Darrieus turbine as a low speed wind energy converter." Engineering Science and Technology, an International Journal 18, no. 1 (2015): 1-13. https://doi.org/10.1016/i.jestch.2014.08.002 
[29] Mohamed, M. H., G. Janiga, E. Pap, and D. Thévenin. "Optimal blade shape of a modified Savonius turbine using an obstacle shielding the returning blade." Energy Conversion and Management 52, no. 1 (2011): $236-242$. https://doi.org/10.1016/i.enconman.2010.06.070

[30] Yang, Hua, Wenzhong Shen, Haoran Xu, Zedong Hong, and Chao Liu. "Prediction of the wind turbine performance by using BEM with airfoil data extracted from CFD." Renewable Energy 70 (2014): 107-115. https://doi.org/10.1016/i.renene.2014.05.002

[31] Khan, Sher Afghan, Musavir Bashir, Maughal Ahmed Ali Baig, and Fharukh Ahmed Ghasi Mehaboob Ali. "Comparing the effect of different turbulence models on the CFD predictions of NACA0018 airfoil aerodynamics." CFD Letters 12, no. 3 (2020): 1-10. https://doi.org/10.37934/cfdl.12.3.110

[32] Zainal, S. T. J. S., C. Tan, C. J. Sian, and T. J. Siang. "ANSYS simulation for Ag/HEG hybrid nanofluid in turbulent circular pipe." Journal of Advanced Research in Applied Mechanics 23, no. 1 (2016): 20-35.

[33] Abobaker, Mostafa, Sogair Addeep, Lukmon O. Afolabi, and Abdulhafid M. Elfaghi. "Effect of Mesh Type on Numerical Computation of Aerodynamic Coefficients of NACA 0012 Airfoil." Journal of Advanced Research in Fluid Mechanics and Thermal Sciences 87, no. 3 (2021): 31-39. https://doi.org/10.37934/arfmts.87.3.3139

[34] ANSYS, ICEM CFD. "11.0 Tutorial Manual, ANSYS." INC., Pennsylvania (2007). 